\title{
Classical Models of Cepheid Stars
}

\author{
C.Chiosi, \\ Department of Astronomy, University of Padova, Padova, Italy
}

\begin{abstract}
In this paper we summarize recent work on Cepheid stars by Chiosi et al (1992a), who calculated large grids of models at varying mass, effective temperature, mass-luminosity (ML) relation or equivalently underlying evolutionary scheme for intermediate mass stars, i.e. classical models, models with mild core overshoot, and models with large core overshoot, and finally initial chemical composition. The chemical parameters bracket the abundances of the Cepheid stars in the Milky Way and Magellanic Clouds. First, we present the theoretical results limited to the analytical fits of the fundamental period-mass-radius (PMR) relation and the blue and red edges of the instability strip for the fundamental mode and first overtone. Second, we discuss the data converted to magnitudes and colours of the BVRcIc passbands with particular attention to the period-luminosity-colour (PLC) and period-luminosity (PL) relations. Finally, we briefly summarize three studies based on these models, which aimed to explain the shape and width of the observed instability strip (Chiosi et al $1992 \mathrm{~b}$ ), and the main reason for the mass discrepancy of the Cepheid stars (Chiosi et al 1992c) by means of the mass equivalency (ME) method applied to clusters of the Large Magellanic Cloud (LMC). Because the ME method turned out to be sensitive to the distance modulus, the dependence of this on the metallicity has been the subject of the last study by Bertelli et al (1992).
\end{abstract}

\section{Introduction}

In recent years, there have been a considerable number of photometric studies of Cepheids in the field of the LMC and SMC (eg. Caldwell \& Coulson, 1986, and references therein) and there is currently much observational effort being put into the search for, and study of, Cepheids in the rich star clusters of the Magellanic Clouds (eg. Mateo et al 1990a,b; Welch et al 1991). Because the lie at the same distance, the Cepheids in the Magellanic Cloud clusters are basic to two important topics of astronomy, the understanding of pulsation theory itself and stellar evolution theories in general, and the establishment of the cosmic distance scale through the calibration of the PLC relation (Schmidt 1984; Feast \& Walker, 1987; van den Bergh 1989; Madore \& Freedman 1991). Furthhermore, modern observations are done increasingly towards the red using BVRcIc photometry rather than the more traditional BV photometry (see Madore \& Freedman 1991). Most of the theoretical modelling of the Cepheid pulsation rests on the pioneer work of Iben \& Tuggle (1972a,b; 1975) and 
Becker et al (1977). See the reviews by Becker $(1985)$ and Chiosi $(1989,1990)$. However, those calculations covered a limited range of masses, were based on old models for intermediate mass stars, and were made mostly at solar metallicity $(Z=0.02)$. The recent work by Chiosi et al (1992a), who produced large grids of Cepheid models covering the appropriate metallicity range and considering the different possible scenarios for the evolution of intermediate mass stars (see below), has much extended this subject. In addition to this, those models, suitably converted into observable photometric quantities, were used to address a few questions, such as the shape and width of the instability strip (Chiosi et al (1992b), and the cause of the discrepancy between the evolutionary and pulsational mass of the Cepheid stars (Chiosi et al 1992c, Bertelli et al 1992). In this paper we shall briefly touch upon these topics.

\section{The Cepheid Models}

All details concerning the physical assumptions of model calculations (e.g. the definition of the growth rate, the boundary conditions, the coupling between pulsation and convection, the mixing length parameter) can be found in Fox \& Wood (1982) and Chiosi et al (1992a), to whom the reader should refer. It suffices to recall that: i) the abundances adopted in those computations were $Y=0.25$ and $Y=0.30$, and $\mathrm{Z}=0.016, \mathrm{Z}=0.008$, and $\mathrm{Z}=0.004$, corresponding approximately with the solar, $\mathrm{LMC}$, and SMC abundance scales, respectively (eg. Russell \& Bessell 1989);

ii) the opacities for these mixtures were computed using the Los Alamos Opacity Library (Huebner et al 1977). The radiative opacities are supplemented by the molecular contribution (Alexander 1975; Alexander et al 1983) according to the prescription by Bessell et al (1989) and the revision by Wood (1990, unpublished);

iii) over the mass range spanned by the models ( 3 to $12 M_{\odot}$ ), three values of the luminosity were associated to each value of the mass in order tobracket the range of luminosities expected from stellar evolution models with and without convective overshoot;

iv) the luminosities and $T_{e f f}$ 's of the models were transformed into absolute BVRcIc magnitudes and colors by means tables of colors and bolometric corrections as a function of the effective temperature, gravity, and chemical composition. Two sources of colours and bolometric corrections were used: the Green et al (1987) data, otherwise known as the 87 release of the Yale isochrones, and based on theoretical model atmospheres calculated by Buser (1989), and otherwise referred to as the Padova scale. These latter was compared with the observational calibrations of the (V-I)and (B-V)- $\log T_{\text {eff }}$ relations for giants (McWilliam 1990) and dwarfs (Bessell 1979). It turned out that Buser's model colors for gravity $\log g=4.5$ closely agree with the dwarf temperature scale. Similarly, the bolometric corrections for the cepheid models agree with the the bolometric correction scale of Flower (1977). within less than 0.05 magnitudes.

The detailed results of the model cepheid calculations are not given here for the sake of brevity. They can be found in Chiosi et al (1992a). Nevertheless, it it worth recalling that while the blue edge of the instability was easy to identify, the red edge 
was more uncertain. Chiosi et al (1992a) adopted as red edge the $\mathrm{T}_{\text {eff }}$ 's at which the growth rate reached its maximum value. As a result of it the instability strip could be wider than assumed (see Chiosi et al 1992a for more details). The discussion below will be limited to the Padova scale.

From the results of the linear calculations linear relationships have been constructed, which represent the numerical results with a sufficient degree of accuracy. For use in the analytical expressions below, we define the following quantities $L^{\prime}=\log L / L_{\odot}$, $T^{\prime}=\log T_{\text {eff }}, M^{\prime}=\log M / M_{\odot}$, and $P_{i}^{\prime}=\log P_{i}$, where the periods are in days and $\mathrm{i}=0,1$ denote the harmonic under consideration.

Period-Mass-Radius Relation. The theory of stellar pulsation reveals that the mass, radius, luminosity, effective temperature, and period of a Cepheid are ideally related by the relations

$$
P \alpha L^{0.75} T_{e f f}^{-3} M^{-0.5}
$$

In practice, the exponents in equation (1) differ from the ideal values. If we write equation (1) in the slightly more general logarithmic form

$$
P^{\prime}=A+B L^{\prime}+C L^{\prime} L^{\prime}+D M^{\prime}+E T^{\prime}
$$

we get the following equations:

Fundamental

$$
P^{\prime}=12.063+0.668 L^{\prime}+0.027 L^{\prime} L^{\prime}-0.691 M^{\prime}-3.551 T^{\prime}
$$

First Overtone

$$
P^{\prime}=10.219+0.785 L^{\prime}+0.000 L^{\prime} L^{\prime}-0.527 M^{\prime}-3.153 T^{\prime} .
$$

As there is little dependence of the coefficients on the chemical composition, a mean fit to all abundances is given. The term in $L^{\prime} L^{\prime}$ was necessary in order to get a good fit. The rms errors in the fits to $\log P$ are all less than 0.005 .

The Instability Strip in the H-R Diagram: The Blue Edge. Along the blue edge of the instability strip, suitable abundance-dependent expressions relating the effective temperature, luminosity, and mass are:

Fundamental

$$
\begin{gathered}
T_{B E}^{\prime}=[3.99+0.07 Y-0.54 Z]+0.01 M^{\prime}-0.09 L^{\prime}+[0.007+0.004 Y-0.009 Z] L^{\prime} L^{\prime} \\
L_{B E}^{\prime}=[2.111+0.302 Y-2.364 Z]+0.738 M^{\prime}+[0.971+0.211 Y-0.595 Z] P_{o}^{\prime}
\end{gathered}
$$

First Overtone

$$
\begin{gathered}
T_{B E}^{\prime}=[3.86+0.06 Y+0.69 Z]+0.11 M^{\prime}-0.01 L^{\prime}+[-0.015+0.014 Y-0.152 Z] L^{\prime} L^{\prime} \\
L_{B E}^{\prime}=[2.174+0.489 Y-1.748 Z]+0.813 M^{\prime}+[0.877+0.326 Y-2.916 Z] P_{1}^{\prime}
\end{gathered}
$$

The Instability Strip in the H-R Diagram: The Red Edge. Similarly for the red edge we get: 
Fundamental

$$
\begin{gathered}
T_{R E}^{\prime}=[3.95+0.12 Y-0.41 Z]+0.04 M^{\prime}-0.10 L^{\prime}+[0.009-0.005 Y-0.074 Z] L^{\prime} L^{\prime} \\
L_{R E}^{\prime}=[1.913+0.237 Y-2.433 Z]+0.862 M^{\prime}+[0.962-0.037 Y-2.314 Z] P_{o}^{\prime}
\end{gathered}
$$

First Overtone

$$
\begin{gathered}
T_{R E}^{\prime}=[3.79+0.15 Y+0.09 Z]+0.06 M^{\prime}-0.01 L^{\prime}+[-0.006-0.006 Y-0.081 Z] L^{\prime} L^{\prime} \\
L_{R E}^{\prime}=[2.088+0.349 Y-1.447 Z]+0.746 M^{\prime}+[1.075-0.174 Y-2.067] P_{1}^{\prime}
\end{gathered}
$$

The $P-M-M_{V}-(B-V)$ Relation. This relation is the analog of the MPLC relation of equation (1). Translated into observational quantities it becomes:

Fundamental

$$
\begin{aligned}
P^{\prime}+0.691 M^{\prime}= & -\left(0.549+0.007 Z_{1}\right)-\left(0.335-0.009 Z_{1}\right) M_{V} \\
& +\left(1.107+0.798 Z_{1}^{2}\right)(B-V)-\left(0.063+0.677 Z_{1}^{2}\right)(B-V)^{2}(13)
\end{aligned}
$$

First Overtone

$$
\begin{aligned}
P^{\prime}+0.527 M^{\prime}= & -\left(0.749+0.004 Z_{1}\right)-\left(0.310-0.006 Z_{1}\right) M_{V} \\
& +\left(1.294+0.904 Z_{1}^{2}\right)(B-V)-\left(0.307+0.938 Z_{1}^{2}\right)(B-V)^{2}(14)
\end{aligned}
$$

The maximum rms error in any of the fits is 0.0071 and the maximum deviation of any point from any of the fits is 0.022 .

The PLC Relation. The PLC (and PL) relation can be obtained from the MPLC relation above with the aid of a ML relation provided by stellar evolution theory. Since the ML relation is a function of the amount of convective overshoot assumed on the main sequence and chemical parameters, the PLC relation will also exhibit such dependences. The ML relation of classical models (without convective overshoot) can be derived from a number of recent evolution calculations which use input physics similar to those used here for the pulsation calculations, in particular, from models using the Huebner et al (1977) opacity. Recent classical evolutionary are by Castellani et al (1990), Lattanzio (1991), and Alongi et al (1992). To obtain the ML relation for models with convective overshoot on the main sequence, an increment $f_{o v}$ in $\log L$ can be simply added to ML relation of classical models. Clearly, $f_{o v}=0$ for classical models, while $f_{o v}=0.25$ is appropriate for models with mild overshoot models and $f_{o v}$ $=0.5$ corresponds to models with full overshoot. We note that $f_{o v}$ is approximately equal to the parameter $d_{\text {over }} / H_{P}$ of Maeder \& Meynet (1989) or half the parameter $\lambda$ of Bertelli et al (1985). The full ML relation is thus

$$
L^{\prime}=-0.015+3.14 Y-10.0 Z+3.502 M^{\prime}+f_{o v} .
$$

Writing the PLC relation in the usual form

$$
M_{V}=\alpha \log P+\beta(B-V)+\phi
$$


we find for the fundamental mode

$$
\alpha=\frac{-5.31}{\left[1+0.115 L^{\prime}\right]},
$$

and

$$
\beta=\frac{\left[\left(110.01+22.81 Z_{1}\right)-\left(28.58+5.92 * Z_{1}\right) T^{\prime}+18.86 /\left(1+0.115 L^{\prime}\right)\right]}{\left[\left(81.1+0.54 Z_{1}\right)-20.4 T^{\prime}\right]},
$$

where, $Z_{1}=\log (Z / 0.016), T^{\prime}=\log T_{\text {eff }}$ and $L^{\prime}=\log (L / L \odot)$. The coefficients $\alpha$ and $\beta$ depend on abundance either explicitly or implicitly through the dependence of $L^{\prime}$ and $T^{\prime}$ on abundance. For Cepheids in the SMC and LMC, Caldwell \& Laney (1991) find $\alpha=-3.75$ and -3.72 and $\beta=2.63$ and 2.45 , respectively, for ridge line solutions. Taking values of $T^{\prime}=3.7$ and $L^{\prime}=3.9$, which correspond to values near the middle of the period range for the LMC and SMC data (ex. Caldwell \& Coulson 1986), and a metal abundance of $\mathrm{Z}=0.008$ for the $\mathrm{LMC}$ and $\mathrm{Z}=0.004$ for the SMC, equations (17) and (18) give $\alpha=-3.67$ for both the LMC and SMC and $\beta=3.16$ in the SMC and 3.12 in the LMC. The observed and theoretical values of $\alpha$ agree well but the observationally determined value of $\beta$ is significantly smaller than the theoretical value. We note, however, that theoretical determinations of $\beta$ generally lie in the range $3.1 \pm 0.1$ (Stothers 1988 ; Stift 1990) and that an analysis of LMC observational data by Stift (1990) also finds a value $\beta \approx 3$. In order to obtain zero points for the PLC relations, and to derive PL relations, a different fit of the theoretical data has been tried in which the explicit dependence on the composition and overshoot parameter are included in the coefficients $\alpha, \beta$, and $\phi$. The coefficient $\alpha$ is assumed to vary as

$$
\alpha=a_{1}+a_{2} Y+a_{3} Z_{1}+a_{4} f_{o v}
$$

and similarly for $\beta$ and $\phi$ (see Table 13 of Chiosi et al 1992a). For LMC and SMC abundances ( $Y=0.27$ and $Z_{1}=-0.3$ and -0.6 , respectively) and no overshoot, $\alpha=-$ 3.69 and $\beta=3.46$ for the LMC and $\alpha=-3.68$ and $\beta=3.59$ for the SMC. The $\alpha$ values agree well with the observed ones and the analytic values derived above. However, the $\beta$ values are larger than the observed or analytic values. It appears that the determination of $\beta$ is quite sensitive to the sample of Cepheids used in the analysis and the method used. We note that Fernie (1990) was unable to find any color term in a PLC relation for Galactic Cepheids. The zero point of the PLC relation provides a test of the amount of overshoot occurring during the main sequence evolutionary phase of Cepheid stars. The procedure works as follows. The zero points of the PLC relation is obtained for abundances appropriate for the Galaxy, the LMC and the SMC, and for no overshoot $\left(f_{o v}=0.0\right)$ and full overshoot $\left(f_{o v}=0.5\right)$. The zero points are then determined from observational PLC relations of the Galaxy, the LMC and the SMC, assuming distance moduli of 18.5 and 18.9 for the LMC and SMC, respectively. These distance moduli are within a few hundredths of the values derived by Caldwell \& Laney (1991) using three separate methods. Finally, the theoretical and observational zero points are compared. The analysis indicates that 
for the Galactic Cepheids zero to mild amounts of convective overshoot $\left(f_{o v}<0.25\right.$ or $d_{\text {over }} / H_{P}<0.25$ or $\left.\lambda<0.5\right)$ seem to be appropriate. For the LMC and SMC, the theoretical and observed values agree best with no overshoot. The final point to be considered regarding the PLC relation is the abundance dependence of the zero points. This can be well represented by the relation

$$
\delta M_{V}=2.15 \delta Y-0.72 \delta \log Z .
$$

For abundances in the range solar to $1 / 4$ solar (Galactic to SMC) these abundance dependences are similar to those given by Stothers (1988).

The PL Relation. The theoretical results indicate that the PL relation for models along the blue edge is abundance independent, while that for the red edge models is only slightly affected. In particular, abundance effects are small compared to changes that result from plausible changes in the amount of overshoot.

In order to compare the observed and theoretical PL relations, some estimate of the way in which Cepheids populate the instability strip is required. Here we assume a uniform population, so that the mean theoretical PL relation lies midway between the blue and red edge sequences. The theoretical PL relation therefore depends on having a good estimate of the red edge, as well as of the blue edge. The theoretical results show that the width of our instability strip is not underestimated by more than $\approx 20 \%$ and that the blue edge is in good agreement with observations, at least for the Galactic Cepheids. The comparison of the theoretical PL relations with the observational ones for the Galaxy (Wilson et al 1991) and for the SMC and LMC (Caldwell \& Laney 1991, and assumed distance moduli of 18.9 and 18.5, respectively) leads to the following conclusions: the theoretical PL relation defined by models with no overshoot is $\approx 0.35$ magnitudes too bright. The theoretical and observational PL relations agree only with mild to large amounts of overshoot.

Limiting the discussion to the case of the fundamental mode and writing the PL relation in the form

$$
M_{V}=A \log P+B
$$

where the coefficients $A$ and $B$ are assumed to have the functional dependence

$$
A=A_{1}+A_{2} Y+A_{3} Z+A_{4} f_{o v}
$$

and are calculated for the mean PL relation defined to be mid-way between the red and blue edge relations. The coefficients are: $A_{1}=-3.092, A_{2}=-0.019, A_{3}=7.799$, $A_{4}=0.003, B_{1}=-1.595, B_{2}=1.018, B_{3}=-4.057$, and $B_{4}=0.702$.

\section{A Few Selected Topics and Conclusions}

Shape of the Instability Strip. The blue edges of the instability strips of Chiosi et al (1992a) agree with the corresponding ones of Iben \& Tuggle (1972a,b; 1975), whereas the red edges have a different inclination whose slope varies with the metallicity. Red edges not running parallel to the blue ones have been suggested by Fernie (1990) for 
the galactic Cepheids and are perhaps confirmed by the observational study of Mateo et al (1990a and references) of Cepheids in LMC clusters. Chiosi et al (1992b) compared the empirical instability strip by Fernie (1990) with the theoretical predictions from the Chiosi et al (1992a) Cepheid models, and by means of the synthetic CMD technique showed that both the edges and the distribution of stars within the strip could be reproduced. In particular, they found that the number frequency-period distribution of Cepheid stars can be better accounted for by adopting models with overshoot.

Mass Discrepancy of the Cepheid Stars. It has long been debated whether the masses determined from stellar evolution theory agree with those derived from pulsation theory (see Iben 1974; Iben \& Tuggle 1972a,b; 1975; Cox 1980, 1985). In general, pulsational masses $\left(M_{p u l}\right)$ are estimated to be $30 \%$ to $40 \%$ lower than evolutionary masses $\left(M_{\text {evol }}\right)$ of the same luminosity. Various causes have been proposed to solve the mass discrepancy problem, each of which would affect the masses in question in a different way (see the reviews by Becker 1985; Cox 1980, 1985; Pel 1985). In the following we would like to call the attention on the role played by convective overshoot as a possible candidate. As already amply discussed, convective overshoot alters the ML relation of core He-burning models. Thus, at any given initial mass, the tracks cross the instability strip at higher luminosity than classical models, or conversely, at any given luminosity the correspondent Cepheid mass is significantly lower (Matraka et al 1982; Bertelli et al 1985). This topic has been examined in a great detail by Chiosi et al (1992c) using the Cepheid stars and CMD of the LMC cluster NGC 2157 (Mateo et al 1990a,b). In fact, the star clusters of LMC with Cepheids are the ideal workbench because all stars lie at the same distance and membership is less of a problem compared to the case of Galactic clusters. On the one hand, the fit of the CMD with theoretical simulations based either on classical models or models incorporating core overshoot leads to accurate determination of the $M_{\text {evol }}$ of the Cepheid stars, together with the age and chemical compositions. In particular, $M_{\text {evol }}$ will turn out to be a function of the underlying distance modulus. On the other hand, the use of the MPLC for Cepheid stars with the chemical composition suited to the cluster in question, allows a good determination of $M_{p u l}$. Once again $M_{p u l}$ is a sensitive function of the underlying distance modulus. By imposing that $M_{\text {evol }}=M_{\text {pul }}$, one may solve for the distance modulus. The study of the Cepheid stars of NGC 2157 indicated that only using models with convective overshoot the distance modulus could agree with other independent estimates. Specifically, the distance modulus to LMC turned out to be $(m-M)_{o}=18.5 \pm 0.1$ in agreement with the recent determination by Panagia et al (1991) based on the circumstellar ring observed by HST around the supernova 1987A in the LMC. When classical models were used, the resulting distance modulus was unacceptably too high. In general, the analysis clarified that the problem of the mass discrepancy likely originates from the adoption of classical models, i.e. without overshoot, to derive $M_{\text {evol }}$, and from the lack of sufficient accuracy in the determination of the distance which bears on both $M_{p u l}$ and $M_{e v o l}$. 
The Distance Modulus to the LMC. The above method (thereinafter the mass equivalency method, ME) has been applied by Bertelli et al (1992) to study the Cepheid stars in two other rich clusters of the LMC, NGC 1866 and NGC 2031, to constrain the cluster distances and the sensitivity to the metallicity. Using evolutionary models incorporating a mild amount of core and envelope overshooting along with the present pulsational models, they derived distance moduli of $18.51 \pm 0.21$ and $18.32 \pm 0.20$ for NGC 1866 and NGC 2031, respectively. The quoted errors are dominated by the uncertainties in the heavy element abundances of the clusters (assumed to be $0.3 \mathrm{dex}$ for both clusters), with a smaller contribution due to the apparently intrinsic spread in the masses of the Cepheids in each cluster. For the ME method, they found that $\Delta(m-M)_{0} / Z_{1}=0.69$, where $Z_{1} \equiv \log (Z / 0.016)$. This result implies that the cluster distances can be determined to better than $\pm 5 \%$ if the cluster abundances can be measured to better than $\sim \pm 0.15$ dex. The distance moduli derived for NGC 1866 and NGC 2031 are consistent with other recent results for the LMC, meaning that the models used in their analysis avoid the classical evolutionary/pulsational Cepheid mass discrepancy. The results of Bertelli's et al (1992) study were based on models using the Los Alamos opacities; had the authors used models incorporating the new Livermore opacities instead, the cluster distance moduli would be larger by at most 0.1 mag.

Radiative Opacities. Studies of the effects of the new radiative opacities by Iglesias \& Rogers (1991a,b) and Rogers \& Iglesias (1992) on the pulsational properties of the Cepheid stars are under way. Preliminary test calculations done for intermediate mass stars with solar composition indicate period variations less than $4 \%$ (Capitanio et al 1992).

\section{References:}

Alongi, M., Bertelli, G., Bressan, A., Chiosi, C., Fagotto, F., Greggio, L., Nasi, E., 1992, Astron. Astrophys. Suppl. in press.

Alexander, D. R., 1975, Astrophys. J. Suppl. 29, 363.

Alexander, D. R., Johnson, H. R., Rympa, R. C., 1983, Astrophys. J. 273, 773.

Becker, S. A., 1985, in Cepheids: Theory and Observations, ed. B. F. Madore, Cambridge University Press, p. 104.

Becker, S. A., Iben, I. Jr., Tuggle, R. S., 1977, Astrophys. J. 218, 633.

Bertelli, G., Bressan, A., Chiosi, C., 1985, Astron. Astrophys. 150, 33.

Bertelli, G., Bressan, A., Chiosi, C., Mateo, M., Wood, P. R., 1992, Astrophys. J. submitted.

Bessell, M.S., 1979, Pub. Astron. Soc. Pacific 91, 589.

Bessel, M. S., Brett, J. M., Scholz, M., Wood, P. R., 1989, Astrophys. J. Suppl. 77, 1.

Buser, R., 1989, private communication.

Caldwell, J. A. R., Coulson, I.M., 1986, Mont. Noc.Roy. Astr. Soc. 218, 223.

Caldwell, J.A.R., Laney, C.D., 1991, in The Magellanic Clouds, ed. R. Haynes and D. Milne, Kluwer, Dordrecht, p. 249.

Capitanio, N., Chiosi, C., Wood, P.R., 1992, in preparation.

Castellani, V., Chieffi, A., Straniero, O., 1990, Astrophys. J. Suppl. 74, 463. 
Chiosi, C., 1989, in The Use of Pulsating Stars in Fundamental Problems of Astronomy, ed. E. G. Schmidt, Cambridge University Press, p.19.

Chiosi, C., 1990, in Confrontation between Stellar Pulsation and Evolution, ed. C. Cacciari and G. Clementini, Astr. Soc. of Pacific Conf. Series 11, p. 158.

Chiosi, C., Wood, P. R., Capitanio, N., 1992a, Astrophys. J. Suppl. in press.

Chiosi, C., Wood, P.R., Bertelli, G., Bressan, A., 1992b, Astrophys. J. $387,320$.

Chiosi, C., Wood, P.R., Bertelli, G., Bressan, A., Mateo, M. 1992c, Astrophys. J. 385, 205.

Cox, A.N., 1980, Ann. Rev. Astron. Astrophys. 18, 15.

Cox, A. N., 1985, in Cepheids: Theory and Observations, ed. B. F. Madore, Cambridge University Press, p. 126.

Feast, M.W., Walker, A.R., 1987, Ann. Rev. Astron. Astrophys. 25, 345.

Fernie, J.D., 1990, Astrophys. J. 354, 295.

Flower, P.J., 1977, Astron. Astrophys. 54, 31.

Fox, M.W., Wood, P.R., 1982, Astrophys. J. 259, 198.

Green, E.M., Bessel, M.S., Demarque, P., King, C.R., Peters, W.L. 1987, in The Revised Yale Isochrones and Luminosity Functions. Yale University, Observatory, New Haven.

Huebner, W.F., Mertz, A.L., Magee, N.H. Jr, Argo, M.F., 1977, Astrophysical Opacity Library, UC-34b.

Iben, I. Jr., 1974, Ann. Rev. Astron. Astrophys. 12, 215.

Iben, I. Jr., Tuggle, R.S., 1972a, Astrophys. J. 173, 135.

Iben, I. Jr., Tuggle, R.S., 1972b, Astrophys. J. 178, 441.

Iben, I. Jr., Tuggle, R.S., 1975, Astrophys. J. 197, 39.

Iglesias, C., Rogers, F.J., 1991a, Astrophys. J. 371, 408.

Iglesias, C., Rogers, F.J., 1991a, Astrophys. J. 371, L73.

Lattanzio, J.C., 1991, Astrophys. J. Suppl. 76, 215.

Madore, B. F., Freedman, W. L., 1991, Publ. Astron. Soc. Pacific 103, 933.

Maeder, A., Meynet, G., 1989, Astron. Astrophys. Suppl. 210, 155.

Mateo, M., Olszewski, E., Madore, B.F., 1990a, Astrophys. J. 107, 203.

Mateo, M., Olszewski, E.W., MadoreB.F. 1990b, in "Confrontation between Stellar Pulsation and Evolution", ed. C. Cacciari and G. Clementini, Astr. Soc. of Pacific Conf. Series 11, p. 241.

Matraka,B., Wassermann, C., Weigert, A., 1982, Astron. Astrophys. bf 107, 283.

McWilliam , A., 1990, Astrophys. J. Suppl. 74, 1075.

Panagia, , N., Gilmozzi, R., Macchetto, F., Adorf, H. M., Kirshner, R. P., 1991, Astrophys. J. $380, \mathrm{~L} 23$.

Pel, J. W., 1985, in Cepheids: Theory and Observations, ed. B. F. Madore, Cambridge University Press, p. 1.

Rogers, F.J., Iglesias, C., 1992, Astrophys. J. Suppl. in press.

Russell, S.C., Bessell, M.S., 1989, Astrophys. J. Suppl. 70, 865.

Schmidt, E. G., 1984, Astrophys. J. 285, 501.

Stift, M.J., 1990, Astron. Astrophys. 229, 143.

Stothers, R.B., 1988, Astrophys. J. 329, 712.

van den Bergh, S, 1989, Astron. Astrophys. Rev 1, 111.

Welch, D. L., Mateo, M., Cote', P., Fisher, P., Madore, B., 1991, Astron. J. 101, 490.

Wilson, T.D., Barnes, T.G., Hawley, S.L., Jefferys, W.H., 1991, Astrophys. J. 378, 708. 